\title{
Neue Elemente und Ephemeride des Kometen 1913 b (Metcalf).
}

Die in A. N. 4682 mitgeteilten Elemente habe ich unter Beihilfe meines Sohnes, stud. $A$. Kobold, durch Anschluß der Beobachtung Bothkamp Sept. 24 und unter Berücksichtigung von Aberration und Parallaxe verbessert. Die Positionen für Sept. 2 u. so wurden durch Hinzuziehung von Beobachtungen zu Mt. Hamilton, bezw. Bergedorf und Taschkent gesichert. Das erhaltene parabolische Elementensystem stellt die Beobachtungen Sept. 2 und 10 streng dar und läßt in dem Ort Sept. 24 die Fehler B $-\mathrm{R}$ übrig:

$$
\Delta \lambda \cos \beta=+5 . \mathrm{I} \quad \boldsymbol{A} \beta=+5.2 \text {. }
$$

Die Elemente nebst Äquatorealkonstanten sind:

$$
\begin{aligned}
& T=\text { I9r } 3 \text { Sept. r } 4 \text {. ror66 n. Z. Berlin } \\
& \omega=\text { I I } 7^{\circ} 4 \mathrm{I}^{\prime} \text { I } 9^{\prime \prime} 5 \\
& \delta=15724 \text { 21.1 } 1913.0 \\
& i=143224.5 \\
& \log q=0.132374 \\
& x=[9.988278] \cdot r \cdot \sin \left(v+46^{\circ} 9^{\prime} \text { 1 } 8.8\right) \\
& y=\left[9.75^{2449}\right] \cdot r \cdot \sin \left(v+\mathrm{I} 5^{6} \text { I } 43^{6.0}\right) \\
& z=[9.932473] \cdot r \cdot \sin \left(v+1275^{8} 40.6\right)
\end{aligned}
$$

\begin{tabular}{|c|c|c|c|c|c|c|c|c|c|c|c|}
\hline 1913 & M. Orts-Zeit & $\Delta \alpha$ & $A \delta$ & Vgl. & $\mathrm{Bb}$ & $\alpha$ app. & $|\log p \cdot \Delta|$ & $\delta$ app. & $\log p \cdot \Delta$ & Red.ad l.app. & * \\
\hline
\end{tabular}

Ephemeride für $\mathbf{I}^{\mathrm{h}} \mathrm{m}$. Z. Berlin, berechnet von $A$. Kobold.

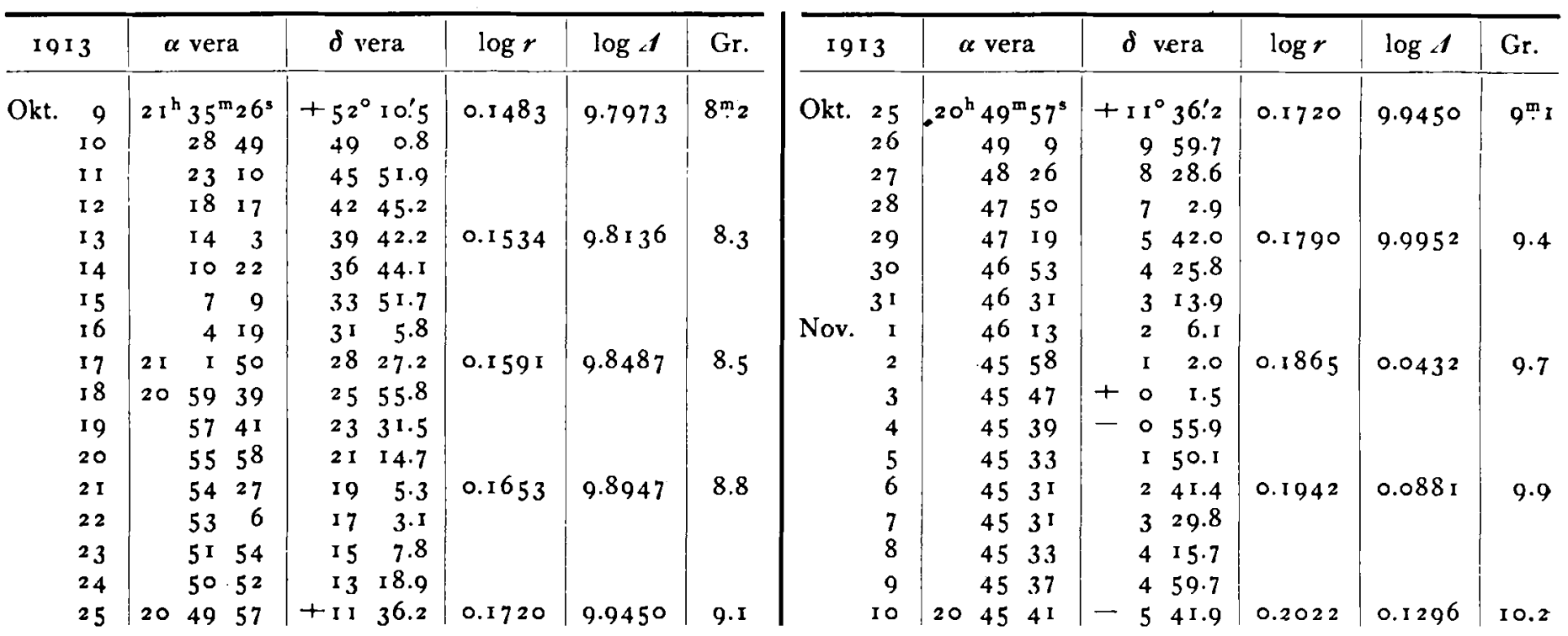

Kiel, Bureau der Astronom. Nachrichten, I 9 r 3 Okt. 7 .

H. Kobold.

Beobachtungen des Kometen 1913 c (Neujmin).

Auf der Sternwarte Utrecht von Prof. A. A. Nijland und Observator F. v.d. Bilt.

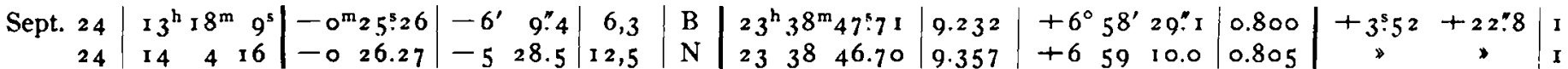

Vergleichstern I: $\alpha$ I9I3.0 $=23^{\mathrm{h}} 39^{\mathrm{m}} 9^{\mathrm{s}} .45 \quad \delta$ I9I3.0 $=+7^{\circ} 4^{\prime}$ I $5^{\prime \prime} 7 \quad$ Lpz II II $74^{6}$.

Auf der Universitätssternwarte Kopenhagen von Prof. E. Strömgren und Assistent R. Andersen.

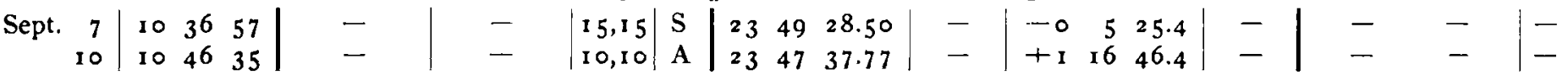

Bei der-geringsten Fadenbeleuchtung erscheint der Komet als punktförmiger Stern i r. Größe, bei vollständig dunklem Felde und dunklen Fäden ist eine sehr schwache Nebelhülle unzweifelhaft sichtbar. Beide Abende genau dasselbe Aussehen.

Telegramm aus Pulkowo vom 8. Oktober: "Dubjago Kasan benachrichtigt: Brightness Neujmin's comet appears fluctuating. Banachiewicz.*

Inhalt zu Nr. 4686. A. Prey. Bemerkungen zu Hansens * Theorie der Sonnenfinsternisse*. 97. - L. Schupmann. Das Medial-Fernrohr zu Landstuhl. ro1. - Mitteilungen uber Kleine Planeten. 105. - H. Kobold. Neue Elemente und Ephemeride des Kometen 1913 b. (Metcalf). I I I. - Beobachtungen des Kometen $1913 \mathrm{c}$ (Nerimin). I I1. 